This item was submitted to Loughborough's Research Repository by the author.

Items in Figshare are protected by copyright, with all rights reserved, unless otherwise indicated.

\title{
Driver discomfort in vehicle seats: effect of changing road conditions and seat foam composition
}

PLEASE CITE THE PUBLISHED VERSION

http://dx.doi.org/10.1016/j.apergo.2015.03.010

PUBLISHER

(C) Elsevier

VERSION

AM (Accepted Manuscript)

\section{PUBLISHER STATEMENT}

This work is made available according to the conditions of the Creative Commons Attribution-NonCommercialNoDerivatives 4.0 International (CC BY-NC-ND 4.0) licence. Full details of this licence are available at: https://creativecommons.org/licenses/by-nc-nd/4.0/

\section{LICENCE}

CC BY-NC-ND 4.0

\section{REPOSITORY RECORD}

Mansfield, Neil J., George M. Sammonds, and Linh Nguyen. 2019. "Driver Discomfort in Vehicle Seats: Effect of Changing Road Conditions and Seat Foam Composition”. figshare. https://hdl.handle.net/2134/17051. 
DOI: 10.1016/j.apergo.2015.03.010

Article accepted for publication: 10 Mar 2015

\title{
APPLIED ERGONOMICS
}

\section{DRIVER DISCOMFORT IN VEHICLE SEATS - EFFECT OF CHANGING ROAD CONDITIONS AND SEAT FOAM COMPOSITION}

\author{
Neil Mansfield \\ George Sammonds \\ Linh Nguyen \\ Environmental Ergonomics Research Centre \\ Loughborough Design School \\ Loughborough University \\ LE11 3TU \\ UK \\ Corresponding author: n.j.mansfield@lboro.ac.uk
}




\section{HIGHLIGHTS}

- Exposure to whole-body vibration accelerates the onset of discomfort when driving

- Improvements in the chemical composition of a polyurethane foam improve the feelings of comfort in a seat when driving for 40 minutes and exposed to vibration

- Cessation of vibration exposure caused an acute improvement in feelings of comfort in a car seat, but discomfort continued to develop with prolonged sitting. 


\section{Abstract}

Discomfort in vehicle seats is a multi-factorial problem with contributions occurring from effects of sitting duration, seat design, and the dynamic environment to which the occupant is exposed. This paper reports laboratory studies investigating the extent to which reports of discomfort are affected by vibration commencing or ceasing, and whether methods of assessment are sensitive enough to detect small changes in foam composition. Study 1 measured discomfort ratings for two conditions of 60 minutes each, comprising 30 minutes of vibration exposure followed by 30 minutes of static sitting in a car seat, and vice-versa. Study 2 measured discomfort ratings for three conditions over a period of 40 minutes each, whilst participants were sitting in one of two car seat compositions, and either exposed to vibration or not. In both studies participants operated a driving simulator. It is shown that exposure to vibration increases the rate of discomfort onset in comparison to periods of static sitting. When vibration stopped, there was an acute improvement in comfort but discomfort did not drop to the levels reported by those who had been unexposed. When vibration started after 30 minutes of static sitting, there was an acute increase in discomfort but not to the levels reported by those who had been exposed to 30 minutes of vibration. After 40 minutes of continuous exposure it was possible to detect significant differences in overall discomfort between the two seat compositions, although trends could be observed in less time. 


\subsection{Introduction}

Inevitably, a proportion of the readers of this paper will be doing so whilst travelling, perhaps on a train, aircraft, bus, or being driven by their chauffeur. Some of them might be facing a long journey ahead, during which time they might become conscious of feelings of discomfort. All types of vehicular travel involve a finite duration of movement as the vehicle travels from origin to destination. Depending on basic properties of average speed, route taken and distance travelled, the duration of the movement would typically range from a few minutes to a few hours. In some cases (e.g. work vehicles; vehicles with on-board accommodation such as some ships or trains) the duration of travel could last significantly longer.

Many factors could contribute to the feelings of comfort whist sitting in a vehicle seat. Ebe and Griffin (2000a, b) produced a model of seat discomfort comprising 'static' and 'dynamic' factors. The foundation of the model was the concept that some factors were a result of the overall design of the seat and did not change rapidly, such as stiffness, pressure distribution and shape, but other factors (dynamic) were caused by the instantaneous dynamic environment (vibration) which the seat was experiencing. There could be an intentional trade-off between the static factors and dynamic factors such that some seats could be optimised dynamically and some statically, although large differences in seat design do not always result in significant improvements in vibration exposure (e.g. Jonsson et al. 2014). An additional dimension of the causes of seat discomfort is the time for which an individual has been seated. Previous studies (e.g. El Falou et al., 2003; Porter et al., 2003; De Carvalho and Callaghan, 2011, Smith et al. 2015) have shown analytically that sensation of overall discomfort increases over time. Through a series of studies involving motion and long-duration sitting, Mansfield et al. (2014) showed that discomfort increases both with vibration magnitude and with sitting duration, and that the presence of vibration causes an increase in the rate of discomfort 
onset. A regression model was proposed that allowed for projection of future overall discomfort depending on short-term ratings of seat discomfort, duration of sitting, and the vibration magnitude.

Whilst previous research has shown differences in discomfort ratings between test conditions, the differences between test parameters have tended to be simplified. For example, where different seat designs have been considered, the differences between the designs have been relatively great, such as using seats from different classes of vehicle, therefore changing several design parameters at once. Where motion has been a key independent variable, the characteristics of the motion have usually been kept constant within a trial, rather than replicating the variations in motion that would occur when driving on different road surfaces during a journey or replicating intermittent motion such as could occur in traffic or on public transport. This paper reports two related studies that consider (a) the effects of changing the motion environment half way through a long-term discomfort test, and (b) the effects of changing the composition of the seat construction.

\subsection{Methods}

This paper reports results from two repeated measures studies using similar equipment, seats and protocols. Studies were conducted at the Environmental Ergonomics Research Centre, Loughborough University. Both studies were approved by the University Ethics Committee, participants provided informed consent, and a pre-trial health screening questionnaire was administered. Participants were required to be aged between $18-65$ and to hold a full UK driving licence to ensure posture and task required during the study would be familiar.

The first study investigated the effect of changing the magnitude of vibration mid-trial; the second study aimed to determine whether the method used was sensitive enough to detect differences between two identically shaped seats but with different foam composition. 


\subsection{Driving Rig}

A rig was built on a six degree-of-freedom multi-axis vibration simulator (MAViS) with closed loop control. It comprised a driving package replicating the dimensions from a current production car. The corresponding car seat was mounted in the rig, as was a representative steering wheel and pedals that were used to control a driving simulator (Fig. 1). Subjects were free to adjust the seat into a comfortable driving position before the start of the trials. The seat had its fabric cover removed such that the foam was in direct contact with the clothing of the subjects. This was to ensure that effects of foam composition (Study 2) were not masked by the presence of the seat cover.

Figure 1 about here.

A pre-recorded vibration file that had been previously measured in a car driving on a rough road was used to produce motion from 1-20 Hz. Vibration on the surface of the seat was calibrated during a system characterisation procedure whereby Biometrics accelerometers were placed on the seat surface in a standard flexible disc and the simulator gain adjusted to provide the desired vibration magnitude prior to the start of the experiment. This equalisation was completed for each participant on each visit to the laboratory. Pilot work showed high degrees of system stability during a trial; the calibration required was less than $10 \%$ in all cases. Where vibration was present, the magnitude was set to $0.5 \mathrm{~m} / \mathrm{s}^{2}$ r.m.s. (root sum of squares, $0.22 x, 0.33 \mathrm{y}, 0.31 \mathrm{z}$, weighted according 
to ISO 2631-1), typical of driving on a moderately rough road. The vibration file was a loop of a 3 minute measurement, which ensured a constant exposure during the vibration conditions.

\section{$\underline{2.2 \text { Discomfort Scores }}$}

Discomfort ratings were collected for both body part and overall discomfort, using two separate rating scales. Body part discomfort was collected using a body map and utilising a 6-point discomfort scale with verbal anchors taken from ISO 2631-1 (1997), with 1 being 'not uncomfortable' and 6 'extremely uncomfortable' (Figure 2). These data were primarily designed as priming questions for the overall rating of discomfort which was elicited using an adapted Borg CR-100 scale (Borg and Borg, 2002; Figure 2). Subjects were trained in the use of the scales before starting the experiments.

Figure 2 about here.

\subsection{Study 1}

In Study 1, participants sat on Foam A, a mass-produced lightweight foam which is used in typical mid-range production cars. Participants were required to drive in a simulation including light traffic. The route comprised driving on highway (motorway), urban, and rural roads. Directions were given via a simulation of a navigation system. Before the trial participants were given 5-minutes practice time without vibration to familiarise themselves with the simulator. 
Ten healthy males were recruited from Loughborough University. Participants' range (mean and \pm s.d.) for age, height and weight were as follows: $22-38$ years old (mean age $28.9 \pm 5.6$ ), $170-195$ $\mathrm{cm}$ (mean height $180.8 \mathrm{~cm} \pm 7.1$ ) and $69-124 \mathrm{~kg}$ (mean $83.74 \mathrm{~kg} \pm 15.6$ ), respectively. Participants attended the laboratory on two different occasions to complete two experimental conditions:

A $(0,0.5) \quad 60$ minutes of driving: Foam A - no vibration over time $0-30$ minutes; continuous vibration over time 30-60 minutes.

$A(0.5,0) \quad 60$ minutes of driving: Foam A - continuous vibration over time $0-30$ minutes; no vibration over time 30-60 minutes.

Notation for each condition is presented with a reference to foam type followed by parenthesis containing and the order of presentation of vibration conditions. E.g. A $(0,0.5)$ refers to foam $A$ exposed to zero vibration followed by vibration at $0.5 \mathrm{~m} / \mathrm{s}^{2}$ r.m.s.

Participants were assigned a random order to complete the trial conditions to minimise order effects. One participant dropped out of the study leaving full data for $n=9$.

Periodically, participants were required to stop driving and complete the discomfort questionnaires. Discomfort ratings were recorded at $0,2,10,20,30,32,40,50$, and 60 minutes.

\subsection{Study 2}

In Study 2, participants sat on each of 2 different foam types. Foam A, as used in Study 1, and an additional foam; Foam B. Foam B had been designed with a special resin structure to have good damping performance, particularly in the roll direction. Participants were again required to perform driving tasks on a driving simulator that consisted of highway (motorway), urban and rural roads. However, in this study participants were required to perform some simple 'follow driving' tasks 
whereby participants were required to follow the vehicle in front along a standardised route. Participants were again allowed 5-minutes practice prior to completing the trial.

Twelve participants were recruited for Study 2 (6 male, 6 female) from the local and student population of Loughborough University. Participants range (mean \pm s.d.) for age, height and weight were as follows: $20-33$ years old (mean age $20.2 \pm 3.5$ ), $158.5-187.5 \mathrm{~cm}$ (mean height $173.6 \pm 9.7$ ) and $54-86.3 \mathrm{~kg}$ (mean weight $69.4 \pm 11.8$ ), respectively. Participants attended the laboratory on three different occasions to complete three experimental conditions:

A (0) 40 minutes of driving: Foam A - No vibration.

A (0.5) 40 minutes of driving: Foam A - With continuous vibration.

$B(0.5) 40$ minutes of driving: Foam B - With continuous vibration.

Notation for each condition is presented with a reference to foam type followed by parenthesis vibration condition. E.g. B (0.5) refers to foam B exposed to vibration at $0.5 \mathrm{~m} / \mathrm{s}^{2}$ r.m.s.

Participants were again assigned a random order to complete the trials to minimise order effects. One participant dropped out of the study leaving full data for $n=11$.

Periodically participants were required to stop driving and provide discomfort ratings. These ratings were collected at 10, 20, 30 and 40 minutes.

\subsection{Results}

\subsection{Study 1}

During the first 30 minutes, the onset of discomfort was more rapid for condition $A(0.5,0)$, the condition with vibration; similarly for the second 30 minutes, the onset of discomfort was more rapid for condition $A(0,0.5)$, the condition with vibration (Figure 3 ). For those periods where there was no vibration, the rate of discomfort onset was less rapid than those periods where there was 
vibration. At the end of the trial there was a mean score of 26.7 (s.d. 12.3) for condition A $(0,0.5)$ and 20.6 (s.d. 10.9) for condition A $(0.5,0)$. In both cases, the verbal anchor corresponded to 'moderate discomfort' for the mean score. Immediately after the vibration stopped for condition A $(0.5,0)$, the overall discomfort rating reduced indicating a decrease in overall discomfort. At the same time for condition $A(0,0.5)$, the vibration started and a step increase in discomfort was shown in the overall discomfort score.

Figure 3 about here.

\subsubsection{Effects of Time}

To investigate the effects of time, paired t-tests compared the overall discomfort rating at 0 and 60 minutes for each condition. There was a significant difference in discomfort between the beginning and the end of the 60-minute trials for both protocols ( $p<0.05$, two-tailed).

Figure 3 shows a trend towards a difference in discomfort at 30 minutes and 60 minutes. Mean discomfort for conditions $\mathrm{A}(0,0.5)$ and $\mathrm{A}(0.5,0)$ were 10.1 and 17.9 at 30 minutes, but 14.4 and 14.4 at 32 minutes respectively. Despite the trends in the data, there was no significant difference at 30 or 60 minutes ( $p=0.12$, t-test two-tailed 30 minutes; $p=0.10$, t-test two-tailed 60 minutes).

\subsubsection{Effects of Vibration}

For the periods with no vibration there was a mean increase of 6.1 for condition $A(0,0.5)$ ( 2 vs. 30 minutes), and 6.7 for condition $A(0.5,0)$ (32 vs. 60 minutes). For the periods with vibration there was a mean increase of 12.2 for Condition A $(0,0.5)$ ( 32 vs. 60 minutes), and 15.3 for condition A $(0.5,0)$ ( 2 vs. 30 minutes). Thus there was less increase in discomfort for the vibration in the second half of the trial compared to the first half of the trial. 
A regression analysis of the time intervals 2 to 30 and 32 to 60 minutes shows high $R^{2}$ values for times with vibration (>0.95), but this dropped to 0.63 when there was no vibration in the second period (Table 1). The gradient for periods with no vibration was $0.23 \mathrm{~A}(0,0.5)$ and $0.19 \mathrm{~A}(0.5,0)$, and $0.46 \mathrm{~A}(0,0.5)$ and $0.55 \mathrm{~A}(0.5,0)$ for periods with vibration.

Table 1 about here

\subsection{Study 2}

The results from Study 2 show that across all conditions, discomfort accrues with time (Figure 4). A clear difference is observed between the two foam types as the onset of discomfort was more rapid for condition $A(0.5)$ and participants recorded lower overall discomfort ratings for condition $B(0.5)$ at the end of the trial. Furthermore, a clear difference is observed when comparing condition $A(0.5)$ and condition $A(0)$, supporting the findings of Study 1 and confirming that discomfort onset is more rapid with exposure to vibration.

Figure 4 about here.

\subsubsection{Effects of Time}


To investigate the effects of time, paired t-tests compared the overall discomfort rating at 10 and 40 minutes for each condition. A significant difference was observed for all 3 conditions $(p<0.005$, twotailed) supporting the findings from Study 1 and implies that discomfort will increase with time regardless of whether vibration is present.

\subsubsection{Effects of Vibration}

T-tests were conducted that compared the discomfort ratings for condition A $(0)$ and the discomfort ratings for condition $A(0.5)$ at each time interval. A significant difference was observed at each time interval ( $p<0.005$, two-tailed) and suggests that discomfort onset will increase significantly with the presence of vibration. This supports the findings from Study 1 and shows that discomfort onset is more rapid with exposure to vibration across the duration of the trial.

\subsubsection{Effects of Foam Composition}

Paired t-tests were conducted that compared the discomfort ratings recorded for condition A (0.5) and the discomfort ratings recorded for condition $B(0.5)$ at each time interval in order to compare between foam types. A significant difference was observed at the 40 minute interval $(p<0.005$, twotailed) implying that, in this case, when comparing the two foam types, a significant difference can only be observed after 40 minutes of exposure.

\subsection{Discussion}

\section{$\underline{4.1 \text { Study } 1}$}

\subsubsection{Effects of Time and Vibration}

The results from this laboratory based simulated driving study, which included a change in vibration protocols, are encouraging in terms of understanding the onset of discomfort in long term driving. 
The results support previous research that has shown how ratings of total discomfort increased significantly during the trial (El Falou et al. 2003; Kyung et al. 2008; Kyung and Nussbaum 2008) and increased more so when vibration was present (Mansfield et al. 2014). After 30 minutes the score for the overall discomfort was more than double that at the start of the trial, indicating that increases in discomfort seem largely associated with fatigue. The rate of discomfort onset was greater when participants were exposed to vibration, compared to participants who were exposed to no vibration. These results are in agreement with El Falou et al. 2003. The presence of vibration amplified the rate at which discomfort increased compared to no vibration. Although the discomfort increased during the trials, the final scores did not reach the extremes of the scales, with maximum mean values equating to verbal anchor scale descriptors of 'Moderate Discomfort' after 60 minutes (Table 2). The maximum value reported by any participant for any trial was 40 , representing the mid-point of the 'High Discomfort' zone. These relatively modest scores parallel those observed in previous studies (e.g. El Falou et al. 2003; Mansfield et al., 2014), where the full scale was not used by participants.

Table 2 about here

The study found that when vibration stopped $(A(0.5,0), 30$ minutes), discomfort immediately decreased showing an acute improvement in conditions. However, the ratings of discomfort did not drop to the levels reported by participants at an equivalent point in condition $A(0,0.5)$; this indicates that there was some retention of the accrued 'discomfort' up to that point. Similarly, when the vibration started after 30 minutes in condition $A(0,0.5)$, the acute increase in discomfort did not reach the level that had been reached at the equivalent time in condition $\mathrm{A}(0.5,0)$, again showing that there was some accrual of previous experiences. 
The drop in discomfort ratings after cessation of vibration for $\mathrm{A}(0.5,0)$ indicates that simple dose models for vibration discomfort (e.g. vibration dose value, VDV (International Organization for Standardization, 1997)) are not suitable for determining instantaneous discomfort sensations, as it is numerically not possible for the VDV to decrease with increases in time. Other vibration metrics, such as r.m.s., have a decay in the response when vibration ceases, but does not have the property of increasing with increased vibration dose. Therefore existing vibration-only models do not adequately represent the overall discomfort.

The acute changes highlight the potential benefit of taking a break from vibration exposure (i.e. driving) but that simply staying seated does not 'reset' the discomfort back to a pre-exposure state. Vibration is one of many factors that contribute to an overall sensation of comfort in a vehicle, and should be considered alongside the posture, seat design and duration of sitting (Mansfield, 2013).

\subsection{Study 2}

\subsubsection{Effects of Foam Composition}

The main aim of Study 2 was to compare the two foam types. The results show that a significant difference is only observed at the 40 minute interval. This implies that in this study, a minimum of 40 minutes testing was required to successfully determine the difference in overall seat discomfort when comparing different foam types. This study normalised for the differences in vibration magnitude experienced on the seat surface; previous work has shown that a difference of approximately $13 \%$ is required to perceive a difference in vibration for road-type stimuli (Mansfield and Griffin, 2000).

A difference of approximately 4 rating points was observed on the discomfort scale after 40 minutes when comparing the results for the two foams. 40 minutes is a relatively short time period in comparison to previous research that has suggested a duration of 2 hours is required to distinguish between seats (Gyi and Porter, 1999). The difference demonstrates that foam Type B performed 
significantly better than Type A in terms of discomfort ratings and signifying that Type B has better static and dynamics properties (combined). The special cell and resin design of Type B has improved damping performance and the results imply that this led to a reduced increase in overall car seat discomfort ratings. This is different to the study of Porter and Gyi, who did not use vibration in their simulations and therefore differences between the dynamic properties of the seats would not have been apparent. This highlights the importance of testing under dynamic as well as static conditions as recommended by Ebe and Griffin (2000a, b), Mansfield (2005) and Mansfield et al. (2014).

The results suggest that foam composition can have significant implications on people undertaking journeys of long durations ( $>40$ minutes in the conditions tested) as differences in foam design is shown to become increasingly relevant as duration increases. Manufacturers must consider foam composition as this can have a significant influence on reducing the onset of discomfort during long duration journeys.

\section{$\underline{4.3 \text { Local Discomfort }}$}

The results for local discomfort (Table 3) reflect a similar trend to those observed for overall discomfort. It is shown that when analysing local discomfort, differences between the conditions can still be observed and that responses for local discomfort support the findings for overall discomfort. However, the main focus of the local discomfort analysis was to highlight areas of particular discomfort and ensure that any increase in overall discomfort could not be attributed to one body region alone.

Table 3 about here 
As reported by Morgan \& Mansfield (2014), the most commonly reported side effect of whole body vibration is lower back and neck pain. The results for local discomfort in this study confirm this to be the case, as the largest increase in discomfort was observed in the lower back. Le et al. (2014) also noted concentrations of discomfort in the neck and back, but also in the lower extremities for a study with 12 participants. The study reported here, however, demonstrates a steady increase in discomfort with time across all body regions and no particular region dominated the local discomfort results.

\subsection{Conclusions}

This paper has shown that there is an acute step change in reported discomfort when exposure to vibration starts or stops. The fatigue effect from long-term sitting and exposure to vehicle motion does not follow the expected responses that would be predicted from vibration-only dose models. Small changes to foam composition were shown to affect the overall discomfort in the seat, but the differences were only significant after 40 minutes. This highlights the importance of long-duration dynamic testing when developing vehicle seats.

\subsection{Acknowledgements}

The seat and foams used in this study were supplied by the Bridgestone Corporation, Japan. 


\subsection{References}

Borg, E., and Borg, G., 2002. A comparison of AME and CR100 for scaling perceived exertion, Acta Psychologica, 109 (2): 157-175.

De Carvalho, D. E., \& Callaghan, J. P. (2011). Passive stiffness changes in the lumbar spine and effect of gender during prolonged simulated driving. International Journal of Industrial Ergonomics, 41(6), 617-624.

Ebe, K., and Griffin, M.J., 2000a. Qualitative models of seat discomfort including static and dynamic factors, Ergonomics, 43 (6): 771-790.

Ebe, K., and Griffin, M.J., 2000b. Quantitative prediction of overall seat discomfort, Ergonomics, 43 (6): 791-806.

El Falou, W., Duchêne, J., Grabisch, M., Hewson, D., Langeron, Y., and Lino, F., 2003. Evaluation of driver discomfort during long-duration car driving, Applied Ergonomics, 34 (3): 249-255.

Gyi, D.E. and Porter, J.M., 1999. Interface pressure and the prediction of car seat discomfort, Applied Ergonomics, 30 (2): 99-107.

ISO 1997, Mechanical Vibration and Shock: Evaluation of Human Exposure to Whole-body Vibration, Part 1, General Requirements: International Standard ISO 2631-1: 1997 (E), ISO.

Jonsson, P.M.G., Rynell, P.W., Hagberg, M., and Johnson, P.W., 2014. Comparison of whole-body vibration exposures in buses: effects and interactions of bus and seat design. Ergonomics, DOI: $10.1080 / 00140139.2014 .961568$

Kyung, G., and Nussbaum, M.A., 2008. Driver sitting comfort and discomfort (part 2): relationships with and prediction from interface pressure. International Journal of Industrial Ergonomics, 38: 526538. 
Kyung, G., Nussbaum, M.A., and Babski-Reevesb, K., 2008. Driver sitting comfort and discomfort (part 1): Use of subjective ratings in discriminating car seats and correspondence among ratings, International Journal of Industrial Ergonomics, 38: 516-525.

Le, P., Rose, J., Knapik, G, and Marras, W.S., 2014, Objective classification of vehicle seat discomfort. Ergonomics 57(4), 536-544.

Mansfield, N. J., \& J Griffin, M. 2000. Difference thresholds for automobile seat vibration. Applied Ergonomics, 31(3), 255-261.

Mansfield, N. J., Mackrill, J., Rimell, A. N., \& MacMull, S. J. (2014). Combined effects of long-term sitting and whole-body vibration on discomfort onset for vehicle occupants. ISRN Automotive Engineering, http://dx.doi.org/10.1155/2014/852607, 2014.

Mansfield, N.J. 2005 Human Response to Vibration. CRC Press.

Mansfield, N.J. 2013 Human response to vehicle vibration, in: Gkikas, N. (Ed.). Automotive Ergonomics: Driver-Vehicle Interaction. CRC Press, Boca Raton, pp. 77-96.

Morgan, L. J., \& Mansfield, N. J. 2014. A survey of expert opinion on the effects of occupational exposures to trunk rotation and whole-body vibration. Ergonomics, 57(4), 563-574.

Porter, J.M., Gyi, D.E., and Tait, H.A., 2003. Interface pressure data and the prediction of driver discomfort in road trials, Applied Ergonomics, 34 (3): 207-214.

Smith, J., Mansfield, N.J., Gyi, D.E., Pagett, M., and Bateman, B., 2015. Driving performance and driver discomfort in an elevated and standard driving position during a driving simulation, Applied Ergonomics, 49, 25-33. 
Figure 1. Laboratory set up including driving rig, seat, steering wheel, pedals and driving simulator.
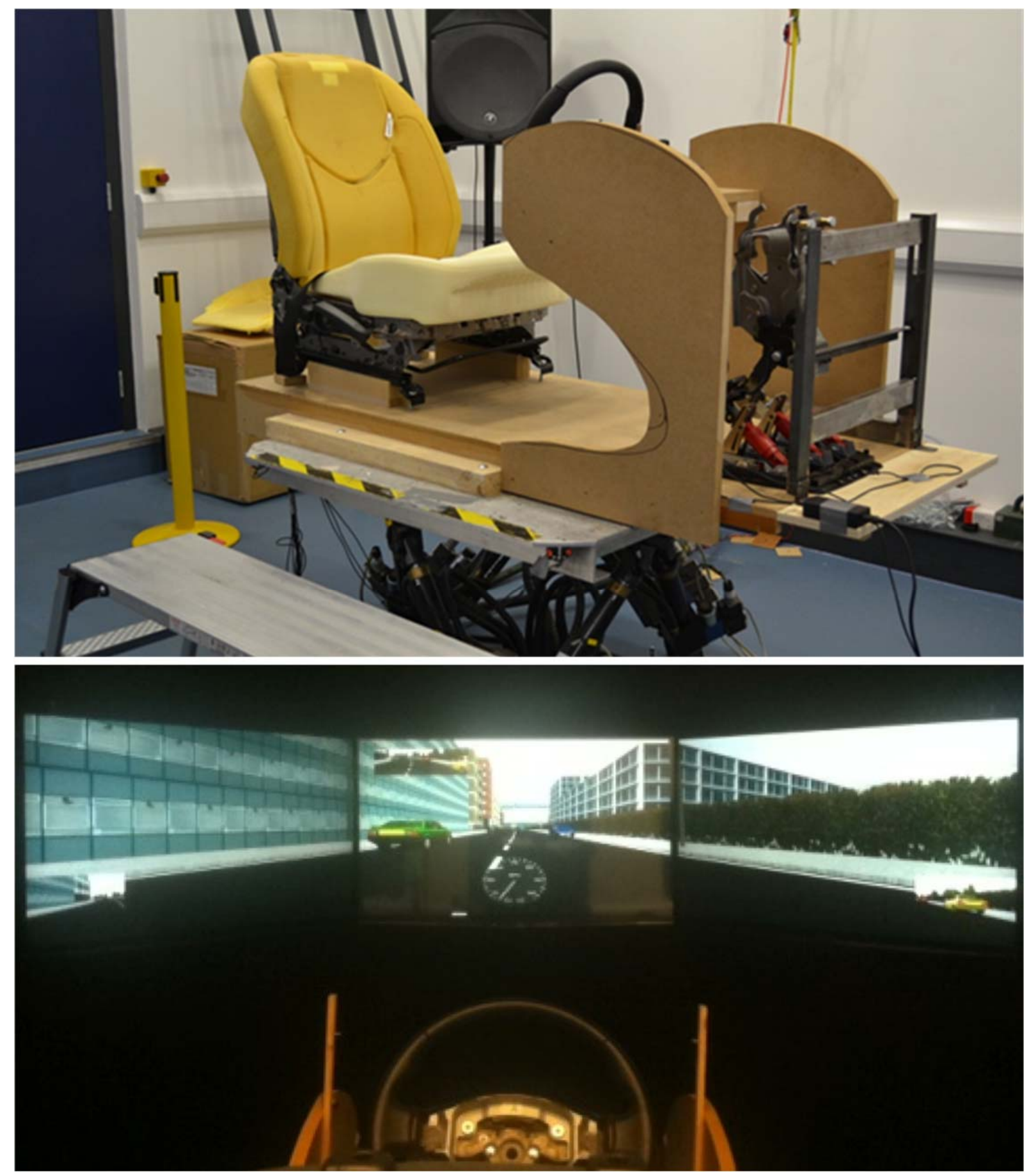
Figure 2. Questionnaire design as used in the experiment. Part 1 focusing on local discomfort, containing the 6 point semantic rating scale as proposed by ISO 2631-1 (1997), and Part 2 focusing on overall discomfort, containing an adapted version of the Borg CR100 scale (Borg \& Borg, 2002).
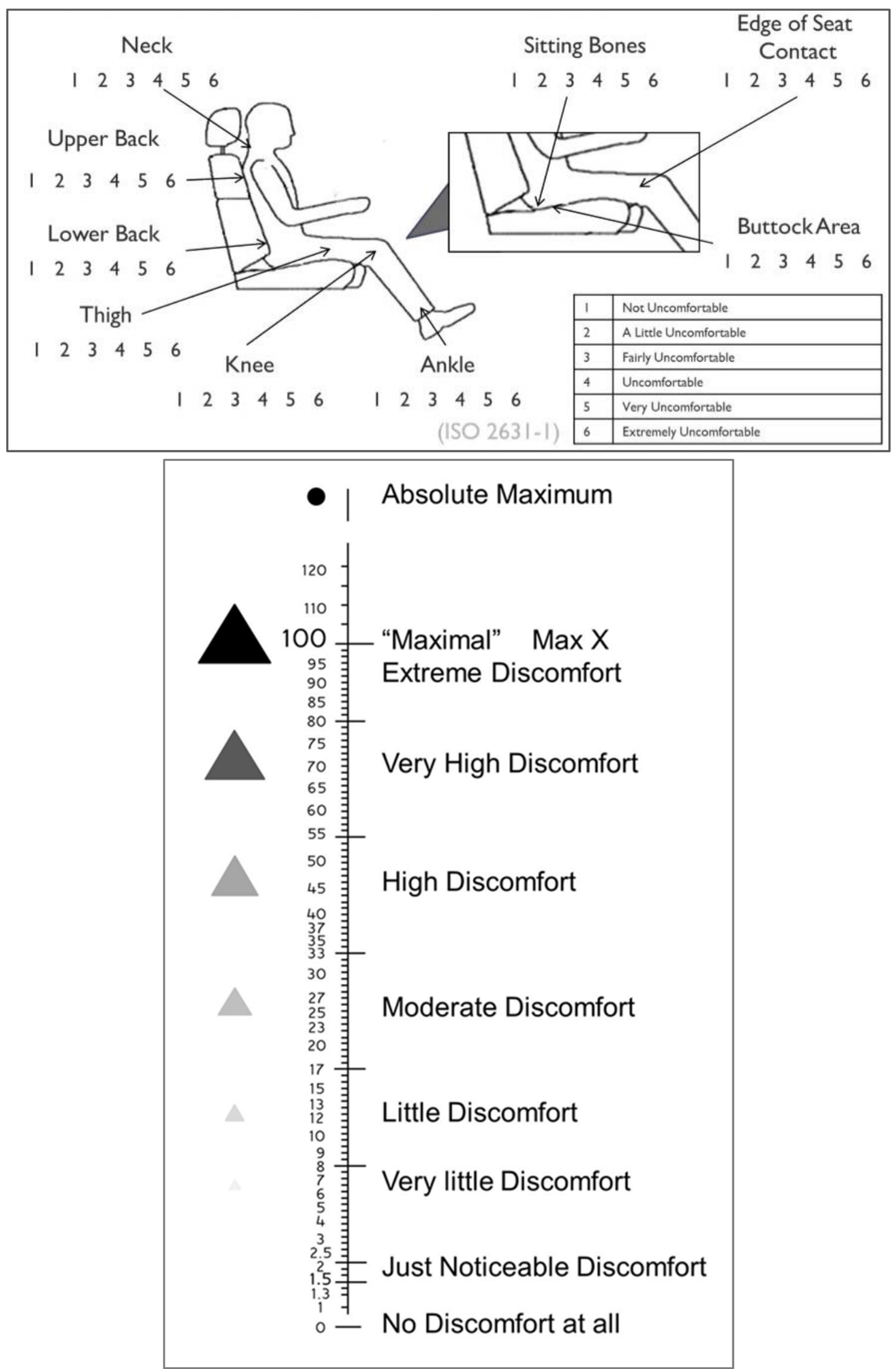
Figure 3. Mean overall discomfort ratings over time for $A(0,0.5)(--\bullet-)$ and $A(0.5,0)(\longrightarrow \bullet)$.

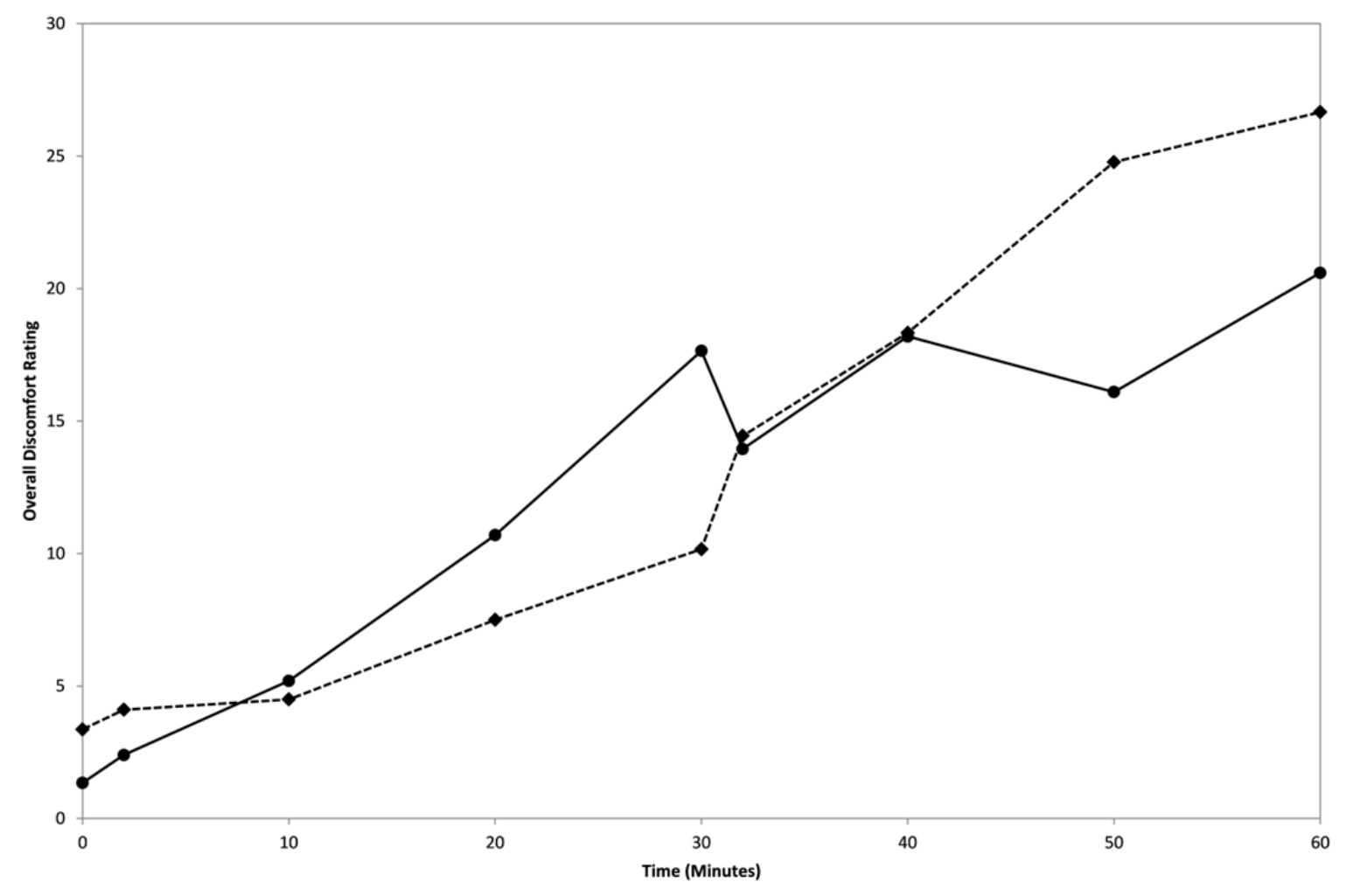


Figure 4. Mean overall discomfort ratings over time for $A(0)(-\square-), A(0.5)(--\diamond--)$ and $B(0.5)$ $(-\Delta \cdot-)$

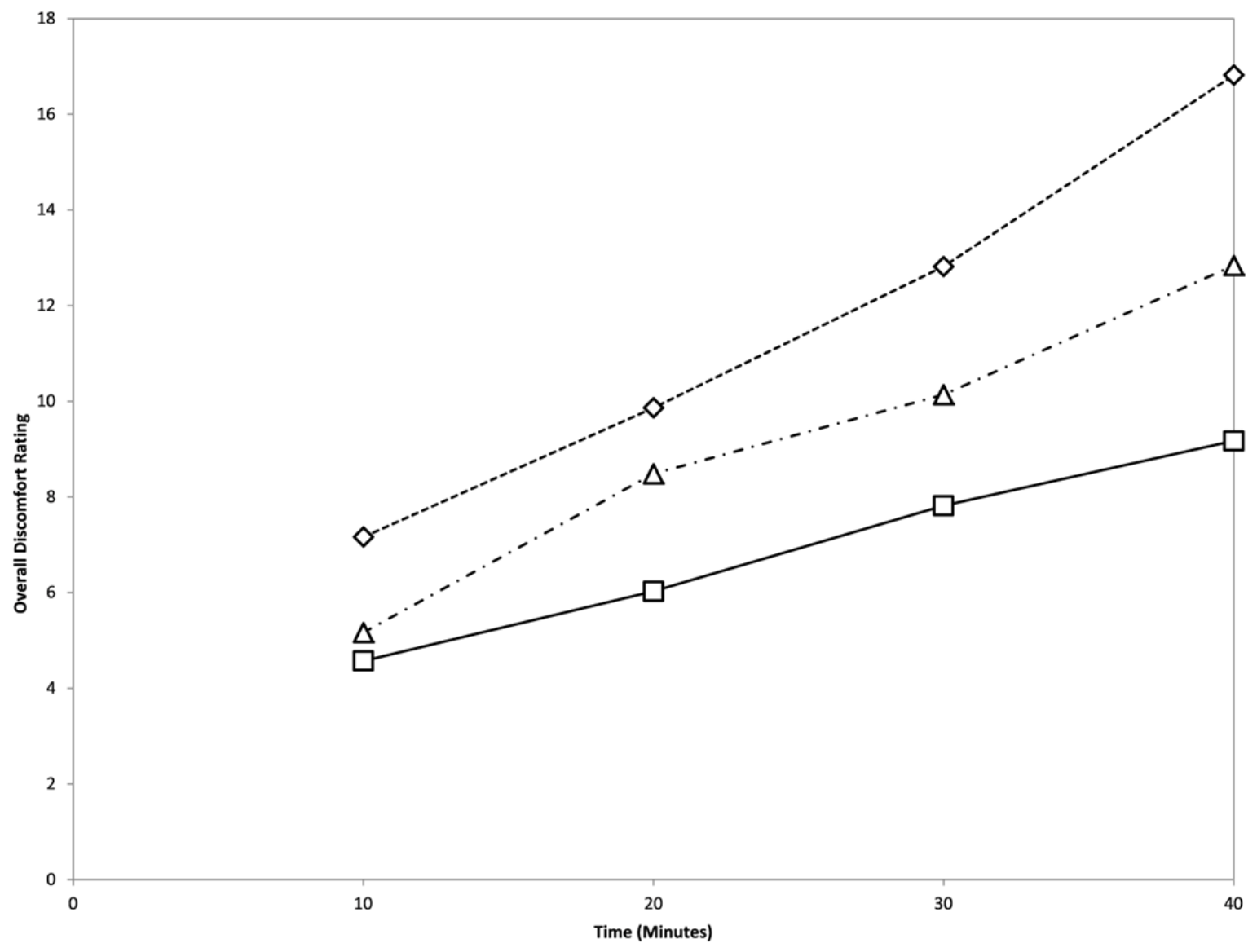


Table 1. Summary of regression equations for discomfort in Study 1.

\begin{tabular}{ccccc}
\hline $\begin{array}{l}\text { Vibration } \\
\text { Protocol }\end{array}$ & $\begin{array}{c}\text { Regression } \\
\text { Equation } \\
(\mathbf{2}-\mathbf{3 2} \mathbf{~ m i n})\end{array}$ & $\mathbf{R}^{\mathbf{2}}$ & $\begin{array}{c}\text { Regression } \\
\text { Equation } \\
\mathbf{( 3 2}-\mathbf{6 0} \mathbf{~ m i n})\end{array}$ & $\mathbf{R}^{\mathbf{2}}$ \\
\hline $\begin{array}{c}\mathrm{A}(0,0.5): \\
\text { Vibration from } \\
30-60 \\
\text { minutes }\end{array}$ & $y=0.23 x+3.04$ & $\mathbf{0 . 9 5}$ & $y=0.46 x+0.27$ & $\mathbf{0 . 9 6}$ \\
\hline $\begin{array}{c}\mathrm{A}(0.5,0): \\
\begin{array}{c}\text { Vibration from } \\
0-30 \\
\text { minutes }\end{array}\end{array}$ & $y=0.55 x+0.48$ & $\mathbf{0 . 9 8}$ & $y=0.19 x+8.72$ & $\mathbf{0 . 6 3}$ \\
\hline
\end{tabular}


Table 2. Summary of mean discomfort scores for all conditions.

\begin{tabular}{|c|c|c|c|c|c|c|}
\hline Condition & $\begin{array}{c}\text { Time } \\
(\mathrm{m})\end{array}$ & Mean & $\begin{array}{l}\text { Standard } \\
\text { Deviation }\end{array}$ & $\begin{array}{l}\text { Conf } \\
(95 \%)\end{array}$ & $\mathbf{N}$ & Scale Descriptor \\
\hline \multirow[t]{9}{*}{$A(0,0.5)$} & 0 & 3.4 & 5.0 & \pm 3.3 & 9 & Very Little Discomfort \\
\hline & 2 & 4.1 & 5.1 & \pm 3.4 & 9 & Very Little Discomfort \\
\hline & 10 & 4.5 & 3.9 & \pm 2.5 & 9 & Very Little Discomfort \\
\hline & 20 & 7.5 & 4.7 & \pm 3.1 & 9 & Very Little Discomfort \\
\hline & 30 & 10.2 & 6.6 & \pm 4.3 & 9 & Little Discomfort \\
\hline & 32 & 14.4 & 9.6 & \pm 6.3 & 9 & Little Discomfort \\
\hline & 40 & 18.3 & 10.9 & \pm 6.5 & 9 & Little - Moderate Discomfort \\
\hline & 50 & 24.8 & 11.6 & \pm 7.5 & 9 & Moderate Discomfort \\
\hline & 60 & 26.7 & 12.3 & \pm 8.0 & 9 & Moderate Discomfort \\
\hline \multirow[t]{9}{*}{$A(0.5,0)$} & 0 & 1.4 & 2.5 & \pm 1.5 & 10 & Just Noticeable Discomfort \\
\hline & 2 & 2.4 & 3.1 & \pm 1.9 & 10 & Very Little Discomfort \\
\hline & 10 & 5.2 & 5.8 & \pm 3.6 & 10 & Very Little Discomfort \\
\hline & 20 & 10.7 & 9.5 & \pm 5.9 & 10 & Little Discomfort \\
\hline & 30 & 17.7 & 11.1 & \pm 6.9 & 10 & Little Discomfort \\
\hline & 32 & 14.0 & 8.7 & \pm 5.4 & 10 & Little Discomfort \\
\hline & 40 & 18.2 & 10.5 & \pm 6.5 & 10 & Little - Moderate Discomfort \\
\hline & 50 & 16.1 & 10.4 & \pm 6.4 & 10 & Little - Moderate Discomfort \\
\hline & 60 & 20.6 & 10.9 & \pm 6.7 & 10 & Moderate Discomfort \\
\hline \multirow[t]{4}{*}{$A(0)$} & 10 & 4.6 & 6.7 & \pm 3.9 & 11 & Very Little Discomfort \\
\hline & 20 & 6.0 & 8.4 & \pm 4.9 & 11 & Very Little Discomfort \\
\hline & 30 & 7.8 & 11.2 & \pm 6.6 & 11 & Very Little Discomfort \\
\hline & 40 & 9.2 & 11.0 & \pm 6.5 & 11 & Little Discomfort \\
\hline \multirow[t]{4}{*}{$A(0.5)$} & 10 & 7.2 & 7.2 & \pm 4.3 & 11 & Very Little Discomfort \\
\hline & 20 & 9.9 & 9.5 & \pm 5.6 & 11 & Little Discomfort \\
\hline & 30 & 12.8 & 12.5 & \pm 7.4 & 11 & Little Discomfort \\
\hline & 40 & 16.8 & 14.6 & \pm 8.6 & 11 & Little - Moderate Discomfort \\
\hline \multirow[t]{4}{*}{ B (0.5) } & 10 & 5.2 & 4.6 & \pm 2.7 & 11 & Very Little Discomfort \\
\hline & 20 & 8.5 & 8.5 & \pm 5.0 & 11 & Little Discomfort \\
\hline & 30 & 10.1 & 9.2 & \pm 5.4 & 11 & Little Discomfort \\
\hline & 40 & 12.8 & 12.0 & \pm 7.1 & 11 & Little Discomfort \\
\hline
\end{tabular}


Table 3. Body region that recorded the highest discomfort rating for each condition at each time interval.

Condition $\begin{aligned} & \text { Time } \\ & (\min )\end{aligned} \quad$ Body Region

\begin{tabular}{|c|c|c|}
\hline \multirow[t]{9}{*}{$A(0,0.5)$} & 0 & Lower Back \\
\hline & 2 & Upper Back, Lower Back and Knee \\
\hline & 10 & Neck \\
\hline & 20 & Lower Back \\
\hline & 30 & Lower Back \\
\hline & 32 & Lower Back \\
\hline & 40 & Lower Back \\
\hline & 50 & Lower Back \\
\hline & 60 & Lower Back \\
\hline \multirow[t]{9}{*}{$A(0.5,0)$} & 0 & Buttock Area \\
\hline & 2 & Upper and Lower Back \\
\hline & 10 & Neck \\
\hline & 20 & Lower Back \\
\hline & 30 & Neck and Lower Back \\
\hline & 32 & Lower Back \\
\hline & 40 & Lower Back \\
\hline & 50 & Lower Back \\
\hline & 60 & Lower Back \\
\hline \multirow[t]{4}{*}{$A(0)$} & 10 & Neck \\
\hline & 20 & Lower Back \\
\hline & 30 & Upper Back \\
\hline & 40 & Upper Back \\
\hline \multirow[t]{4}{*}{$A(0.5)$} & 10 & Lower Back \\
\hline & 20 & Lower Back \\
\hline & 30 & Lower Back \\
\hline & 40 & Lower Back \\
\hline \multirow[t]{4}{*}{ B (0.5) } & 10 & Upper Back \\
\hline & 20 & Neck, Upper Back and Lower Back \\
\hline & 30 & Neck \\
\hline & 40 & Lower Back \\
\hline
\end{tabular}


